\title{
Intrinsically Resistant Bacteria as Looming Disaster: A Rare Case Report of Elizabethkingia meningoseptica Meningitis in a Neonate
}

\author{
Priyadarshini Patro ${ }^{1}$ Padma Das ${ }^{1}$ Phalguni Padhi \\ ${ }^{1}$ Department of Microbiology, All India Institute of Medical \\ Sciences, Raipur, Chhattisgarh, India \\ 2Department of Neonatology, All India Institute of Medical \\ Sciences, Raipur, Chhattisgarh, India \\ Address for correspondence Padma Das, MD, Department of \\ Microbiology, All India Institute of Medical Sciences, Gate No 5, \\ Medical College Building, First Floor, Tatibandh, G. E. Road, Raipur, \\ Chhattisgarh 492099, India (e-mail: drdaspadma@gmail.com).
}

J Lab Physicians 2021;13:70-73.

\begin{abstract}
Elizabethkingia meningoseptica is a recognized cause of neonatal meningitis with high mortality rate of approximately $57 \%$, but clinical data detailing these infections remain limited from India. Though this bacteria has a Gram-negative character, it is usually multidrug resistant to antibiotics usually prescribed for Gram-negative bacterial infections and susceptible to antibiotics for Gram-positive bacteria, thus poses a serious challenge to the treating clinicians. Such cases of neonatal meningitis is most commonly associated with prematurity with birth weight $<2,500 \mathrm{~g}$, but here we report an uncommon case of neonatal meningitis due to this rare pathogen in a full-term neonate with weight as per gestational age. The isolate was multidrug resistant and

Keywords

- Elizabethkingia meningoseptica

- neonatal meningitis

- multidrug resistant

- multidisciplinary interventions discrepancy was seen between disc diffusion and automated antibiotic susceptibility testing for few antibiotics. The case was successfully managed by treatment with combination of piperacillin-tazobactam, vancomycin, chloramphenicol, and rifampicin for a total duration of 28 days, due to prompt identification of the causative organism and initiation of appropriate antimicrobial therapy early. E. meningoseptica can cause severe infection, with risk of high mortality and neurological sequelae in neonates. Intensive care and multidisciplinary interventions are crucial for case management.
\end{abstract}

\section{Introduction}

Elizabethkingia meningoseptica is an ubiquitous Gram-negative nonfermentating bacillus and a rare pathogen infrequently isolated from various clinical specimen. ${ }^{1,2}$ It causes meningitis in neonates with mortality rates of approximately $57 \%$ and less commonly nosocomial pneumonia, sepsis, and meningitis in both immunocompromised adults and neonates. ${ }^{1}$ It usually affects premature infants and often occur as outbreaks. ${ }^{3}$ Although E. meningoseptica infections in immunocompromised hosts are a well-known entity, limited clinical data are available from the Indian subcontinent.

published online

February 22, 2021
DOI https://doi.org/

10.1055/s-0041-1724234 ISSN 0974-2727.
For E. meningoseptica, multidrug resistance is a rule rather than exception and more so for antimicrobials used for Gram-negative infections. It is unique being susceptible to drugs like vancomycin, clindamycin, and rifampin which are used for treating Gram-positive infection. Presently, neither the Clinical and Laboratory Standards Institute (CLSI) nor the European Committee on Antimicrobial Susceptibility Testing has any recommendation for performance and interpretation of antimicrobial susceptibility test (AST) for this rare pathogen. This often leads to inappropriate choice of antibiotics for initial empirical therapy and results in treatment failures and high mortality. ${ }^{2}$

(C2021. The Indian Association of Laboratory Physicians.

This is an open access article published by Thieme under the terms of the Creative Commons Attribution-NonDerivative-NonCommercial-License, permitting copying and reproduction so long as the original work is given appropriate credit. Contents may not be used for commercial purposes, or adapted, remixed, transformed or built upon. (https://creativecommons.org/licenses/by-nc-nd/4.0/).

Thieme Medical and Scientific Publishers Pvt. Ltd. A-12, 2nd Floor, Sector 2, Noida-201301 UP, India 


\section{Case Report}

We report a case of neonatal meningitis in a 13-day-old full-term, outborn male neonate with seizures and respiratory difficulty since birth. He was born by emergency cesarean section at a local hospital in view of meconium stained amniotic fluid and prolonged labor. The neonate was then shifted to some other child care hospital due to respiratory difficulty where he had episodes of seizures and was given ventilatory support, inotropic support, platelet transfusion, intravenous antibiotics (colistin, meropenem), and antifungals. But due to worsening condition, the baby was referred to our institute.

On examination the following parameters were found, weight $3.2 \mathrm{~kg}$, temperature $36.5^{\circ} \mathrm{C}$, pulse rate $160 / \mathrm{min}$, respiratory rate $50 / \mathrm{min}$, regular, with bilateral crepts, and blood pressure 90/54 mm Hg. Cardiovascular and per abdomen examination was normal. On central nervous system examination, the child was dull looking, with increased tone, eyes closed but easily awakable with reflex cry, and positive activity.

The baby was put on improvised continuous positive airway pressure/oxygen support, intravenous fluids, and intravenous piperacillin/tazobactam, cefotaxime, and amikacin started empirically after sending the blood and cerebrospinal fluid (CSF) for routine laboratory investigations (day 0).

Routine hemogram showed hemoglobin\% $12.1 \mathrm{~g} / \mathrm{dL}$, total red blood cell count 3.71 million $/ \mu \mathrm{L}$, total leucocyte count (TLC) 1 $8.2 \times 10^{3} / \mu \mathrm{L}$, differential leucocyte count (DLC) N73L24M02E01, platelet count $147,000 / \mu \mathrm{L}$, and C-reactive protein $139.1 \mathrm{mg} / \mathrm{L}$.

The CSF was slightly turbid with TLC 427 cells $/ \mathrm{mm}^{3}$, DLC N96L02M02, glucose $<3 \mathrm{mg} / \mathrm{dL}$, and protein $167 \mathrm{mg} / \mathrm{dL}$. The CSF Gram stain showed few pus cells and plenty of short slender Gram-negative bacilli. On the next day (day +1 ), chocolate agar showed heavy growth of 1 to $2 \mathrm{~mm}$ smooth, circular, grayish-white colonies ( - Fig. 1), grayish-white, nonhemolytic colonies on blood agar (-Fig. 2), and nonlactose fermenting semitranslucent colonies MacConkey agar (-Fig. 3). The isolate was Gram-negative bacilli, both catalase and oxidase positive and nonmotile. The neonatologist was

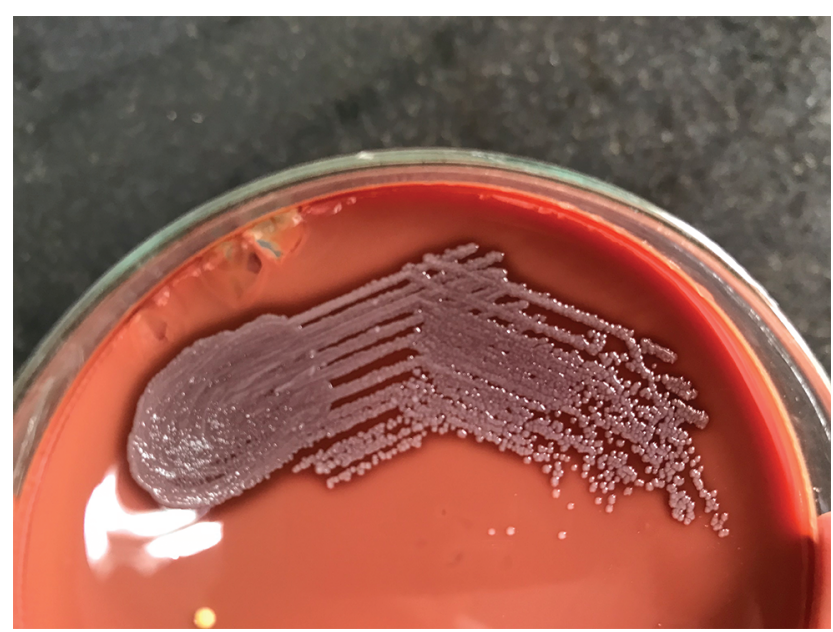

Fig. 1 Growth of smooth, circular, grayish-white colonies on chocolate agar.

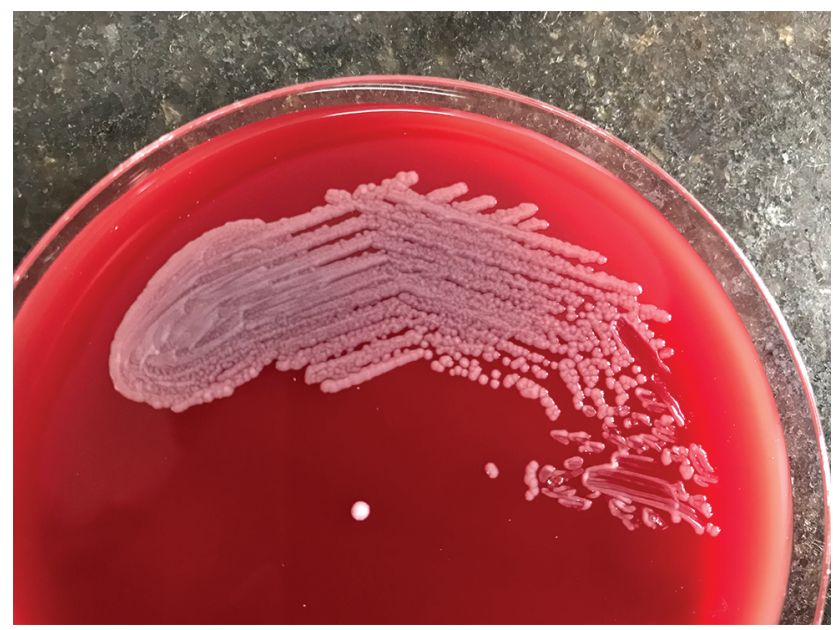

Fig. 2 Growth of smooth, circular, grayish-white, nonhemolytic colonies on blood agar.

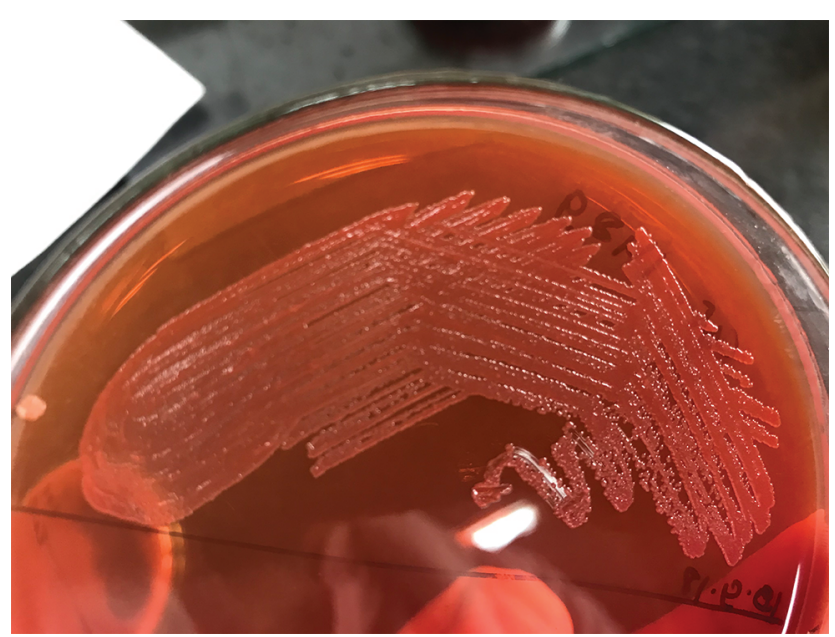

Fig. 3 Growth of smooth, circular, nonlactose fermenting semitranslucent colonies on MacConkey agar.

informed about some unusual nonfermenter with $E$. meningoseptica as one of the differential diagnosis (day +1). So AST was done simultaneously by both Kirby-Bauer disc diffusion method and Vitek 2 automated system. It was identified as E. meningoseptica by Vitek 2 automated system (day +2 ). As CLSI has not established breakpoints for this rare pathogen, the interpretive criteria for non-Enterobacteriaceae and Staphylococcus aureus were used to interpret AST. On Kirby-Bauer disc diffusion test, the bacteria was found to be multidrug resistant, showing resistance to all beta lactams, ampicillin-sulbactam, aminoglycosides, carbapenems, quinolones, chloramphenicol, tetracycline, and rifampicin; but was susceptible to piperacillin-tazobactam, co-trimoxazole, vancomycin, and linezolid. But Vitek 2 AST results showed resistant to most of the antibiotics including piperacillin/ tazobactam (minimum inhibitory concentration [MIC] $\geq$ $128 \mu \mathrm{g} / \mathrm{mL}$ ) and co-trimoxazole ( $\mathrm{MIC}=80 \mu \mathrm{g} / \mathrm{mL}$ ) but susceptible to minocycline only $(\mathrm{MIC} \circledast \leq 1 \mu \mathrm{g} / \mathrm{mL}$ ). Vancomycin MIC was $8 \mu \mathrm{g} / \mathrm{mL}$ (intermediate). The neonatologist was informed about the isolate (day +2$)$ and cefotaxime and amikacin were stopped immediately and vancomycin and ciprofloxacin 
were added to piperacillin/tazobactam. Isolation precautions were followed and strict asepsis was maintained.

The automated blood culture flagged positive on the day +1 and showed short Gram-negative bacilli which later on identified to be the same pathogen, that is, E. meningoseptica, with similar antibiogram.

Intravenous ciprofloxacin was stopped and chloramphenicol was added (day +4$)$. Repeat CSF culture on day +6 again showed E. meningoseptica with the same antibiogram. Following this syrup rifampicin was added to the therapy (day +6$)$.

On day +9 , hemogram showed improvement. On day +14 , CSF and blood culture sent was sterile. The child showed clinical signs of improvement on day +16 . Repeat CSF and blood cultures sent on day +23 were sterile subsequently. Magnetic resonance imaging of brain done on day +25 showed grossly communicating hydrocephalus with diffuse bilateral cerebral white matter edema.

All the four antibiotics were continued for a total duration of 28 days. The child was being discharged on request and parents were counseled on long-term outcomes, regular follow-up, and early intervention. On discharge the child was on oral feeds/breastfeeds, able to maintain temperature without warmer, gaining weight, and vitals stable.

\section{Discussion}

E. meningosepticais a rare cause of meningitis in newborns, mostly associated with premature birth and half of the infections have involved neonates weighing $<2,500 \mathrm{~g}$ and produces severe postinfectious sequelae including brain abscesses, hydrocephalus, deafness, and developmental delay. ${ }^{4}$ In the present case, the patient was a term infant weighing $3.2 \mathrm{~kg}$ and had developed hydrocephalus.

Most of the infections are generally nosocomial and has been associated with immunosuppression, underlying medical illness, prolonged hospital stay, prior exposure to multiple antibiotics, indwelling central venous catheter, and other invasive devices. ${ }^{2,3}$ Several cases of outbreaks of neonatal meningitis have been reported and source was traced to as contaminated water supply, saline, antibiotic solutions, respirators, intubation tubes, incubators for newborns, etc. ${ }^{1,3,5}$ Environmental surveillance should be performed in such cases to identify the source. In our case as the neonate was outborn, it is quite possible that the baby might have acquired the infection in any of the previous two hospitals where he stayed; however, this attribute is purely conjectural as no surveillance was done in the said hospital. But infection control precautions were strictly followed to prevent any outbreak in the neonatal intensive care unit.

Antimicrobial susceptibility data of E. meningoseptica is scarce as it is a rare pathogen. Moreover, results of susceptibility testing vary when different methods (viz., disc diffusion, microbroth dilution, and E-test) are used, ${ }^{4,6}$ and found in the present case also. It is resistant to multiple antibiotics, especially $\beta$-lactams as it produces two $\beta$-lactamases, viz., extended-spectrum $\beta$-lactamase and a carbapenem hydrolyzing metallo- $\beta$-lactamase ${ }^{4}$ which is also seen in our case. But studies have shown that susceptibility of the isolates was relatively high (>50\%) only to piperacillin, piperacillin-tazobactam, trimethoprim-sulfamethoxazole, and ciprofloxacin. ${ }^{3}$ According to a review of medical literature, vancomycin has been recommended for the treatment of Elizabethkingia meningitis, but the efficacy has been questioned in several recent studies, with regards to the high minimum inhibitory concentration $(\geq 16 \mu \mathrm{g} / \mathrm{mL}){ }^{7}$

It is also unclear to what extent in vitro sensitivity predicts clinical outcome when infections by Elizabethkingia spp. are encountered. ${ }^{6}$ Considering these arguments, combination of piperacillin-tazobactam and vancomycin was given initially because of its in vitro sensitivity by disc diffusion. Chloramphenicol and rifampicin was added later because of many reports of successful treatment of $E$. meningoseptica infection with these adjuvant therapy. ${ }^{3,6}$

CSF and blood culture became sterile which shows effective antibiotic therapy with combination, but unfortunately the baby developed hydrocephaly and other late neurophysiological complications are possible over the next several years.

The incidence of $E$. meningoseptica may be underreported because of lack of wide availability of an automated bacterial identification system. It is therefore essential that laboratories should identify the nonfermenters up to species level and awareness among clinicians about this organism as a potential pathogen is the need of the hour.

\section{Conclusion}

E. meningoseptica is an emerging infection and a nosocomial threat, with high risk for complications and mortality among premature neonates, immunocompromised, or critically ill patients, because of its resistance to multiple antibiotics. A high degree of suspicion, rapid diagnosis, and prompt institution of appropriate therapy for prolonged time period $(\sim 3-4$ weeks) are key factors in management of such infections.

\section{Funding}

None.

\section{Conflict of Interest}

There are no conflicts of interest.

\section{References}

1 Bhat KS, Priya R, Krishnan L, Kanungo R. Elizabethkingia meningoseptica bacteremia in a neonate: a case report and mini-review of the literature. J Curr Res Sci Med 2016;2:42-45

2 Tak V, Mathur P, Varghese P, Misra MC. Elizabethkingia meningoseptica: an emerging pathogen causing meningitis in a hospitalized adult trauma patient. Indian J Med Microbiol 2013;31(3):293-295

3 Ghafur A, Vidyalakshmi PR, Priyadarshini K, Easow JM, Raj R, Raja T. Elizabethkingia meningoseptica bacteremia in immunocompromised hosts: the first case series from India. South Asian J Cancer 2013;2(4):211-215

4 Chamalla SK, Karri SS, Koripadu S, Mohan N. Elizabethkingia meningoseptica: an emerging pathogen causing neonatal meningitis. J Dr NTR Univ Health Sci 2018;7(3):213 
5 Hoque SN, Graham J, Kaufmann ME, Tabaqchali S. Chryseobacterium (Flavobacterium) meningosepticum outbreak associated with colonization of water taps in a neonatal intensive care unit. J Hosp Infect 2001;47(3):188-192

6 Issack MI, Neetoo Y. An outbreak of Elizabethkingia meningoseptica neonatal meningitis in Mauritius. J Infect Dev Ctries 2011;5(12):834-839
7 Arbune M, Fotea S, Nechita A, Stefanescu V. Emerging infection with Elizabethkingia meningoseptica in neonate. A case report. J Crit Care Med (Targu Mures) 2018;4(3):96-100 